\title{
EL ESTUDIO DE LA SEXUALIDAD EN JÓVENES ESPAÑOLES
}

\author{
Maria Lameiras Fernandez
}

\begin{abstract}
RESUMEN
El objetivo de este trabajo es el de llevar a cabo una revisión de los estudios encaminados a evaluar la actividad sexual de los jóvenes españoles, haciendo especial referencia a la población universitaria. Este objetivo se articula a partir de la exposición de las principales variables que han centrado el interés de las investigaciones: 1) conocimientos, tanto en relación a la sexualidad en general como a la infección HIV/aids; 2) actitudes, tanto hacia la sexualidad como hacia la infección HIV/aids; 3) percepción de riesgo en relación a la transmisión sexual del HIV/aids; y 4) comportamientos sexuales, incluyendo tanto la información relativa a la actividad sexual coital, el uso de preservativos y otros métodos anticonceptivos. A partir de esta revisión podemos concluir que los/as jóvenes españoles han incrementado sus conocimientos en los últimos anos especialmente en relación a la infección HIV/aids, tienen
\end{abstract}

*Universidad de Vigo - Campus de Ourense

InterAÇÃO, Curitiba, v. 2, p. 133-161, jan./dez. 1998 
actitudes positivas hacia la sexualidad, pero todavía tienen una baja percepción de riesgo en relación a la transmisión sexual del HIV y, aunque se ha incrementado el uso del preservativo, todavia se mantienen conductas de riesgo, tanto para la transmisión sexual del HIV como de embarazos no deseados. Se considera por tanto necesario continuar con el esfuerzo preventivo llevado a cabo en la última década incluido dentro de programas de educación afectivo-sexual que se inicien en los primeros anos de escolarización y en las que se contemple la intervención con las madres y padres.

Palabras clave: sexualidad en adolescentes, conducta sexual, actitudes sexuales.

\begin{abstract}
The objective of this work is to carry out a revision of those studies which are directed to appraise the sexual activity of the Spanish youth, specially referring to the university population. This objective is constituted from the statement of the main variables on which the research works have focused their interest: 1) knowledge in relation to both sexuality in general and the HIV/aids infection; 2) attitudes towards both sexuality and the HIV/aids infection; 3) risk perception in relation to the HIV/aids sexual transmission, and 4) sexual behaviours, including both the information relative to the coital sexual activity and the use of condoms and other birth-control methods. From this revision we can come to the conclusion that in the last few years the Spanish young people have increased their knowledge, specially in relation to the HIV/aids infection; they take positive altitudes towards sexuality but they still have a low risk perception in relation to the HIV sexual transmission; and, although the condom use has been increased they still maintain risk behaviours to both the HIV sexual transmission and the unwanted pregnancy. It is therefore considered the need to continue with the preventive effort carried out in the last decade, included in the affective-
\end{abstract}


sexual education programmes, to be initiated in the first school years and where the intervention with parents is provided.

Key words: adolescenfs sexuality, sexual behavior, sexual attitudes.

\section{INTRODUCCIÓN}

El estudio científico de la sexualidad tiene una corta historia, que se inicia con las alusiones hechas por Freud en sus trabajos ya a finales del siglo XIX y se aviva con las aportaciones de Haverok Ellis en los comienzos del siglo XX. Pero, el interés por el estudio de la sexualidad se ha visto eclipsado por la férrea presión moralista que ha dificultado su expansión y de la que se va desprendiendo muy lentamente. Esto ha propiciado que el abordaje de la sexualidad haya quedado relegada durante este siglo a pinceladas en mayor o menor medida frutíferas, destacando con especial relevancia los trabajos sociológicos de Kinsey (1948, 1953), las aportaciones en el campo de la psicofisiología y la intervención en disfunciones sexuales del tandem formado por Master y Johnson $(1966,1970)$ y las investigaciones de Helen Kaplan $(1974,1979)$ entorno al deseo y la terapia sexual, hasta la eclosión de estudios que se desencadena en la actualidad.

Será la aparición de la infección provocada por el virus de la inmunodeficiencia humana (HIV/aids) cuando en el verano de 1981 se identifica el primer caso, lo que sitúe el estudio de la sexualidad en un insospechado protagonismo que havisto así crecer su interés e influencia. Enfocado hacia el estudio de la identificación de las variables que subyacen a laconductasexual para promovere instaurar un comportamiento sexual saludable, que no implique riesgos para la salud de los sujetos y que cristaliza con la puesta en marcha de campañas de educación sexual. 
El interés que la sexualidad despierta desde la "era del sida (aids)" se ha focalizado en el estudio de los más jóvenes, especialmente los/as adolescentes dirigiendo hacia este colectivo el grueso de las investigaciones que sobre sexualidad se han efectuado. Recurriendo a las encuestas CAPC (conocimientos, actitudes, percepción de riesgo y conducta) que han propiciado la aparición de una amplia documentación en relación a los niveles de conocimientos de los que los más jóvenes disponen, tanto en relación con la sexualidad en general como con la transmisión de la infección HIV/aids; las actitudes sexuales y hacia afectados/as por el HIV/aids; el nivel de percepción de riesgo o vulnerabilidad percibidaal HIV; para finalmente abordar el nivel de conducta sexual, tanto edad de inicio, frecuencia de ejecución y tipo de prácticas como la evaluación de la utilización de métodos anticonceptivos, y especialmente el uso del preservativo.

Es por tanto el objetivo de este trabajo aportar la información sobre la documentación disponible en torno a los grandes temas a los que se ha hecho alusión en el campo de la sexualidad: el nivel de conocimientos, actitudes, percepción de riesgo y comportamientos de los jóvenes adolescentes españoles a través de la reciente proliferación de trabajos de investigación que ha propiciado el interés por el estudio de la sexualidad en España en la era del sida.

\section{LA ADOLESCENCIA}

La adolescencia es un producto cultural de las sociedades desarrolladas que hace referencia a una edad de "tránsito" que se inicia con la pubertad y que finaliza con la incorporación del sujeto al grupo de adultos. Enmarcar cronológicamente esta etapa supone tener en cuenta la variabilidad interindividual, aunque puede considerarse la media de edad de inicio alrededor de los 10,5 años para las chicas y de 11,5 años para los chicos (López y Fuertes, 1990). La O.M.S. 
$(1875,1979)$ recomienda establecer la edad inferior a los 19 anos como edad límite para enmarcar la adolescencia, que se caracteriza por profundos cambios tanto a nivel físico, psicológico como social. Cambios que se inician con la activación del eje hipotalámicohipofisiario-gonadal y la aparición delas características sexuales secundarias, la formación de la identidad personal (Erikson, 1968), junto con el desarrollo de la capacidad de llevar a cabo un razonamiento hipotético-deductivo (Piaget, 1932), para completarse con la transición hacia la independencia marcada fundamentalmente a nivel económico. Esta última condicionada por la capacidad de autoabastecerse, aunque el progresivo aumento de los períodos de instrucción dilatan reiteradamente la adquisición de la independencia de nuestros jóvenes, lo que Moncada (1979) conceptualiza como "adolescencia forzosa".

Todos estos cambios bio-psico-sociales van a permitir la reorientación de la actividad sexual, desarrollada en la infancia con un carácter lúdico y autodirigido: reconocimiento del propio cuerpo, comportamiento autoexploratorio y autoestimulación, hacia un objetivo implícito o fin en sí mismo que será el placer o satisfacción sexual con la incorporación del "otro". La sexualidad adquiere en esta etapa un especial protagonismo concentrando energías y preocupaciones.

\section{CONOCIMIENTOS SOBRE SEXUALIDAD Y ETS}

A pesar de que los estudios sobre el tema han comprobado de forma inequívoca y rotunda que la información no es suficiente para propiciar cambios de conducta que lleve a los sujetos a instaurar estilos de vida saludables, esta constituye un prerrequisito necesario para poder desencadenar una actividad sexual protegida (GonzalezSaiz, Ortega-Marlasca y Salvador, 1994). 


\section{Conocimientos sobre sexualidad}

Inicialmente se hará alusión al nivel de conocimientos fruto de la autoevaluación que hacen los sujetos del mismo. En el trabajo con alumnas/os pre-universitarios de Lameiras y González (1993), al que ya se ha hecho alusión, se les pregunta directamente a los sujetos encuestados si creen que tienen un nivel de conocimientos suficientes sobre sexualidad. Prácticamente la mitad (el 53.5\%) responden negativamente. En el estudio de Oraá (1996), con una muestra de 689 sujetos escolarizados y no escolarizados riojanos con edades comprendidas entre 17 y 20 años, el porcentaje de sujetos que se consideran bien informados en torno a cuestiones sexuales se incrementa al $73 \%$.

Sin embargo, realmente interesante es identificar el nivel real de conocimientos que poseen los sujetos y en todo caso valorar las discrepancias que se puedan dar entre el nivel real versus autopercibido para explicar la motivación a una autoexposición a los mismos, en los casos en los que el nivel sea bajo y autopercibido como tal.

Al analizar realmente cuál es el nivel de conocimientos sobre sexualidad de que disponen se constata la existencia de mitos y falsas creencias que tienen un efecto claramente negativo sobre el desarrollo de una actividad sexual satisfactoria y saludable. Así, en el trabajo de Lameiras y González (1993) el 42\% considera que el orgasmo simultáneo es una señal inequívoca de ajuste sexual de la pareja y un $27 \%$ considera inadecuado mantener relaciones sexuales durante la menstruación, una falsa creencia muy afianzada también en otras culturas. Además un $66 \%$ cree erróneamente que el sexo del feto depende de la madre, un $48 \%$ que la píldora anticonceptiva impide el encuentro del óvulo con el espermatozoide y para el $25 \%$ el líquido expulsado por el pene, previo a la eyaculación, no puede provocar embarazo. Frente a estos errores, un 63\% reconoce la capacidad poliorgásmica de la mujer y un $60 \%$ que la esterilización 
por vasectomfa no afecta a la satisfacciónsexual del varón. En el trabajo de Aláez, Mayor de la Torre, Madrid, Bavín y Melero (1994), con una muestra de 331 adolescentes madrileños, el $60.2 \%$ de los jóvenes consideran entre las falacias más frecuentemente observadas, que los métodos anticonceptivos disminuyen el placer en las relaciones sexuales, el $51.9 \%$ que no hay días más o menos fértiles en el ciclo femenino y el $48.9 \%$ que la píldora del día siguiente se toma cuando no ha "bajado la regla". Frente a estas se reconoce correctamente por el $91.2 \%$ de los sujetos que el embarazo se produce por la unión del óvulo y el espermatozoide y que el clítoris es un órgano de la mujer.

Así, el nivel de conocimientos en torno a la sexualidad se ha visto mejora do en los últimos años gracias a las campanas de educación sexual que se han puesto en marcha, mayoritariamente a través de campanas informativo/formativas que se han multiplicado en los últimos años. Pero, a pesar del incremento significativo de conocimientos todavía persisten errores; así, en el estudio de Oraá el $26 \%$ de los sujetos creen que en la primera relación sexual la probabilidad de quedarse embarazada una chica es diferente a las veces posteriores, lo que también comprueba Aláez, Mayor de la Torre, Madrid, Bavín y Melero (1994). El 64\% desconoce cuáles son los días fértiles de la mujer, el $13 \%$ cree que la postura del coito determina la probabilidad de embarazo, el $19 \%$ cree que existe relación entre la probabilidad de embarazo y el orgasmo de la chica y el $22 \%$ piensa que éste está condicionado por la profundidad de la penetración. Con una muestra de 547 estudiantes pre-universitarios, Lameiras, Failde y Navas (1997) comprueban cómo solamente el $32.2 \%$ sabe que no es necesario inflar de aire el preservativo antes de ponerlo para comprobar si está pinchado y el 54.9\% que el líquido que se expulsa antes de laeyaculación puede provocar un embarazo, datos equiparables a los obtenidos con una muestra de 72 adolescentes pre-universitarios (Lameiras,1997b). 


\section{Conocimientos sobre si da y otras ETS}

Los esfuerzos educativos dirigidos a incrementar los conocimientos en relación a la infección VIH/sida (Urzelay, Urturi, Elizalde, Zubia y Villafranca, 1991) han permitido que los jóvenes hoy en día dispongan de un buen nivel de conocimientos (Gonzalez-Saíz, Ortega-Marlasca y Salvador, 1994). Así en los diferentes trabajos dirigidos a evaluar el nivel de conocimientos en torno a ETS especialmente el sida, se constata el razonable nivel de información tanto en relación a las vías de infección como a los mecanismos que evitan el contagio (Lameiras, 1997b; Lameiras, Failde y Navas, 1997). Claramente superior al observado en investigaciones anteriores en las que se concluía que el nivel de información es superficial y que los sujetos eran escasamente conscientes de que se puede transmitir la enfermedad en la fase asintomática, con las graves consecuencias que ello tienen para la prevención, a pesar de que conocen la posibilidad de transmisión parenteral y sexual, y el valor profiláctico del preservativo (Failde y González, 1993). En sintonía con estos resultados se pronuncian Lameiras y Gonzalez (1993) quienes comprueban que los conocimientos sobre las ETS son insuficientes y especialmente sobre el sida, constatándose el grave error que desde el punto de vista de la prevención de que la mayoría de los sujetos (86\%) considera que a simple vista puede ser identificado/a una persona portadora de HIV, que pudo haber sido favorecido por las campañas de información en los medios de comunicación que podrían haber provocado el solapamiento entre portador y enfermo (en fase terminal y muy deteriorado físicamente).

Invirtiéndose claramente el porcentaje en investigaciones posteriores en las que se comprueba que el $89.4 \%$ de los sujetos sabe que a simple vista no se puede identificar a una persona infectada por el HIV (Lameiras, Failde y Navas, 1997). En este trabajo con 547 jóvenes pre-universitarios se comprueba que el $86 \%$ de los sujetos sabe que las ETS pueden transmitirse sin que se note ningún 
síntoma, el $90 \%$ que el sida es una enfermedad en la que el organismo se vuelve incapaz de hacer frente a las infecciones y el 89.5\% que para saber si una persona está infectada por el virus del sida debe realizarse un análisis de sangre específico y el $81 \%$ sabe que estar infectado no es igual a padecer la enfermedad. En relación a las vías de contagio, el $94.3 \%$ sabe que el sida no se contagia através de un abrazo y el $98.3 \%$ sabe que el virus del sida se puede contagiar compartiendo agujas o/y jeringuillas para drogarse, al donar sangre una persona infectada $(96.3 \%)$, y si mantiene sexo vaginal (95.9\%). El 94.5\% sabe que usar correctamente el preservativo es la mejor manera de prevenir la infección por el virus del sida a través de las relaciones sexuales con penetración. En la misma linea van los resultados obtenidos por Lameiras (1997b) en el que se comprueba que el $100 \%$ de los sujetos encuestados sabe que el sida puede contagiarse a través de las relaciones sexuales, que para no contagiarse es necesario no utilizar agujas ya usadas y evitar el contacto con sangre de otra persona, además el $97.2 \%$ sabe que para no contagiarse con el $\mathrm{VIH}$ hay que utilizar siempre el preservativo.

\section{ACTITUDES DE LOS/AS ADOLESCENTES HACIA LA SEXUALIDAD Y LAS ETS (SIDA)}

El estudio de las actitudes ha centrado la atención en el campo de la psicología como ningún otro concepto teórico. Identificado como un constructo latente en el que la dimensión valorativa (evaluating) constituye su sena de identidad (Petty, Wegener y Fabrigar, 1997), ha sido definido como la "valoración particular con algún grado de aprobación o desaprobación hacia un objeto de actitud" (Eagly y Chaiken, 1993, p. 1). La utilidad de este omnipresente concepto en la literatura psicológica según Greenwald (1989) viene condicionada porque las actitudes: (1) cumplen determinadas "funciones" para el sujeto, de las que deriva un beneficio psicológico (Katz, 1960; Smith, Bruner y White, 1956); (2) actúan como fuerza selectiva, guía de la percepción y la memoria 
ejerciendo su efecto tanto a nivel de exposición de la información como de percepción, codificación y recuerdo (Pratkanis, Breckler y Greenwald, 1989); y finalmente, (3) por su supuesta capacidad de "predecir" la conducta, aspecto prioritario ya que como nos recuerda Eiser $(1989$, p. 70$)$ "si no somos capaces de predecir lo que la persona hará o no hará en una situación dada ¿qué utilidad tienen nuestras medidas y teorías?"

White, Fisher, Byrne y Kingma (1977), en un intento de sistematización del constructo de actitud hacia la sexualidad y por la necesidad de contar con un instrumento de medida adecuado, llevan a cabo el primer paso elaborando una escala de 21 items llamada Sexual Opinion Survey - revisada y actualizada por Fisher, Byrne, White y Kelley (1988) - mediante la que se intenta ubicar a los sujetos a lo largo de una dimensión de personalidad de erotofobia-erotofilia. Definida como la "disposición aprendida a partir de la exposición particular a las restricciones y castigos relacionados con la sexualidad durante el proceso de socialización" (Fisher, Byrne, White y Kelley, 1988, p. 123).

Fisher, Byrne, White y Kelley (1988) aportan evidencia empírica para defender que las personas identificadas como erotofílicas, es decir, con actitudes positivas hacia la sexualidad se diferencian de las erotofóbicas, personas con actitudes negativas hacia la sexualidad, en que: a) prestan más atención, procesan y recuerdan más información sexual o contraceptiva, b) anticipan en mayor medida la probabilidad de tener relaciones sexuales, c) adquieren en mayor medida métodos anticonceptivos, d) tienen una mayor capacidad para hablar con la pareja sexual sobre el tema de la sexualidad en general y en concreto de las prácticas anticonceptivas, y e) usan de forma más consistente los métodos anticonceptivos.

Estas características constituyen los cinco pasos considerados por Byrne (1983) como necesarios para llevar a cabo una conducta contraceptiva eficaz. De modo que los sujetos erotofílicos están más informados, tienen más habilidades para llevar a cabo conductas que 
no impliquen riesgos en la esfera de la sexualidad, y ejecutan en mayor medida estas conductas. Por tanto la escala nos permite discriminar adecuadamente no solamente en que nivel de la dimensión actitudinal se ubicaría el sujeto con respecto a la sexualidad, sino, y que es más importante, identificar a los sujetos con actitudes erotofóbicas y que, si llevan a cabo conductas sexuales es más probable que se impliquen en mayores riesgos que los erotofílicos. Información relevante para articular los recursos preventivos en materia de salud sexual.

Junto a la Sexual Opinion Survey otras escalas se han desarrollado para medir actitudes sexuales, recurriendo en la mayoría de los casos a escalas tipo Likert (Likert, 1932), y al Diferencial Semántico (Osgood, Suci y Tannembaum, 1957) disponiéndose de un amplio abanico de recursos aunque no siempre validados adecuadamente.

\section{Las actitudes sexuales}

Una "juventud liberal y no traumatizada por el sexo" es la conclusión a la que llegan Fernández, González, González y López (1993, p. 175) en su estudio con una muestra de 400 estudiantes universitarios/as orensanos/as utilizando una escala de actitudes tipo Likert. En el trabajo que en ese mismo año llevan a cabo Lameiras y González (1993, p. 137) con una muestra de 391 estudiantes pre-universitarios, agrupándose la mayoría en el rango de edad 17-18 anos, en relación a las actitudes medidas con una escala tipo Likert y Diferencial Semántico defienden la "moderada aceptación o no rechazo de la homosexualidad, elligero liberalismo y el escasa puritanismo. Rechazo en su mayoría de la promiscuidad sexual y del acceso a la prostitución, valoración de la monogamia e indefinición con respecto a la virginidad"?

Entre las conclusiones obtenidas en los trabajos que hemos efectuado más recientemente y recurriendo a la escala S.O.S., se comprueba la existencia de una juventud erotofílica, para la que bañarse desnudo/a con una persona del otro sexo, ver una película 
de contenido erótico-sexual y masturbarse son las experiencias valoradas más positivamente, al igual que están mayoritariamente de acuerdo en considerar que el material erótico no es algo sucio. También muestran actitudes positivas hacia la homosexualidad ajena aunque rechazan mayoritariamente la posibilidad de tener experiencias homosexuales y las experiencias sexuales en grupo (Lameirasy González, 1996; Lameiras, 1997a; Lameiras y Failde, 1998). Resultados equiparables a los estandarizados a población española obtenidos por Carpintero y Fuertes (1994) (ver tabla 1).

Se confirman las diferencias significativas en función del sexo, siendo los chicos más erotofílicos que las chicas; en función de la opción religiosa, son los creyentes practicantes los que manifiestan actitudes más erotofóbicas hacia la sexualidad y los no creyentes las más erotofílicas; $y$, finalmente, en función de la orientación política con la que se identifican, aunque esta relación no es tan clara como la observada con las variables anteriormente comentadas, son las chicas y chicos identificados con partidos políticos de izquierda nacionalista y aquellas/os que no se identifican con ninguno las/os que muestran actitudes más erotofílicas.

La existencia de diferencias entre sexos relativas a las actitudes sexuales, más permisivas en los varones que en las mujeres, ha sido una de las principales conclusiones en la que convergen diferentes estudios tanto en nuestro país (López, 1990a) como fuera de nuestras fronteras (Baird, 1993; DeSousa y Hutz, 1995). Sin embargo, hay trabajos en los que a pesar de constatarse la existencia de diferencias, éstas parece que tienden a reducirse (López, Lévy, Samson, Frigault, Lamer y Lew, 1993).

En relación a la mayor erotofilia de las personas que se declaran no creyentes, hay que recordar que la vinculación entre actitudes sexuales y opción religiosa no es nueva. Podríamos decir que ha sido una de las variables más vinculadas y justificativas de las actitudes sexuales dentro y 
fuera de nuestras fronteras (Halpern YUdry, 1994; Lévy, Perez y Frigault, 1995; López, Lévy, Samson, Frigault, Lamer y Lew, 1993).

Finalmente la vinculación entre la ideología política y las actitudes hacia la sexualidad se plantea en distintos trabajos. En el estudio de López (1990a) con una muestra de universitarios salmantinos se detecta que a pesar de haber perdido peso desde el estudio de 1977, la ideología política sigue siendo un predictor claramente signiticativo, ya que quienes confiesan tener una ideología de izquierdas mantienen opiniones más liberales, que supone actitudes más positivas hacia la sexualidad, en definitiva más erotofílicas.

\section{TABLA 1: PUNTUACIÓN MEDIA EN ACTITUDES EN FUNCIÓN DEL SEXO MEDIANTE LA ESCALA S.0.S.}

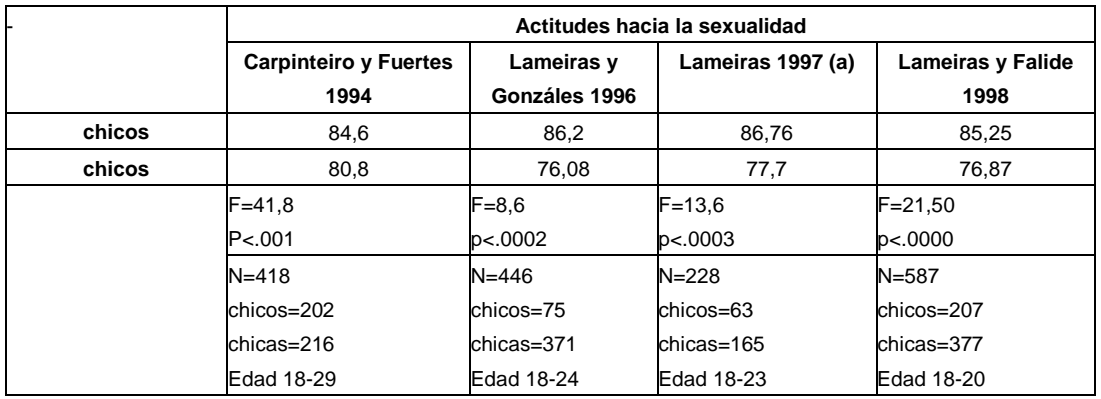

\section{Actitudes de los/as adolescentes hacia el sida}

En el trabajo de Fernández, González, González y López (1993) el $84 \%$ de los alumnos/as universitarios orensanos las interrogados considera que la enfermedad sida es un problema grave, el $81 \%$ tienen miedo y al $85 \%$ les preocupa el contagio. Además un $65 \%$ creen que el sida va a afectar a la elección de sus compañeros/as sexuales y sólo el $55 \%$ de ellos y $44 \%$ de ellas dicen saber cómo protegerse adecuadamente. Si hacemos referencia a la valoración que los sujetos hacen de los/as afectados/as se constata la primacía de posturas no segregacionistas, aunque un $21 \%$ dan respuestas indicativas de 
distanciamiento o prejuicios. Datos que convergen con los obtenidos en otras investigaciones con universitarios españoles (Biascoy Taylor, 1991).

\section{PERCEPCIÓN DE RIESGO}

La percepción de riesgo en relación a la infección VIH/aids supone la valoración personal que lapersona hace de su vulnerabilidad al contagio. A la hora de analizar la percepción de riesgo la forma más habitual de medida ha sido a través de auto-informes en los que a través de preguntas directas se valora el riesgo que el sujeto percibe de contagiarse con el VIH. En el trabajo de Fernández, González, González y López (1993) el 38\% de los sujetos sexualmente activos/as se consideran en riesgo respecto a la infección $\mathrm{VIH} /$ aids y dicha percepción de riesgo se incrementa notablemente en los que no usaron siempre el preservativo y han cambiado de pareja.

Sin embargo una forma alternativa de medida es a través de una metodología indirecta con la que se pretende incrementar la fiabilidad de la información, debido a las dificultades que plantea la metodología directa para evaluar el ámbito de la sexualidad, limitando su validez y fiabilidad. Así Bayés, Pastells y Tuldrá $(1995,1996)$ proponen a través de tres historias (en las que los sujetos encuestados tienen que hacer el esfuerzo de identificarse con el/la (protagonista) la valoración del riesgo de transmisión del virus del aids. Los resultados de las dos investigaciones ratifican que los jóvenes perciben una falsa sensación de seguridad cuando las relaciones sexuales se mantienen con la pareja frente a las relaciones sexuales casuales, lo que se confirma con la investigación de Lameiras (1997a; Lameiras y Failde, 1998). En la figura 1 se representa gráficamente el nivel de percepción de riesgo que perciben las tres muestras independientesde sujetos. La primera muestra identificada como "piloto" la forman 228 alumnos/as del primer curso universitario del campus de Orense en el curso 1995-1996 (el 72.4\% de chicas y 
el $27.6 \%$ de chicos). La segunda muestra está formada por 587 alumnos/as de primer curso universitario del campus de Orense en el curso $1996-1997$ (el $64.6 \%$ de chicas y el $35.4 \%$ de chicos), finalmente la última muestra está formada por 468 estudiantes universitarios de Barcelona durante el curso $1995-1996$ (el $74 \%$ de chicas y el $26 \%$ de chicos).

Los resultados muestran las tendencias equiparables para lastres muestras en relación al nivel de riesgo percibido a las tres historias planteadas. Así en la primera historia, una relación de sexo casual, el nivel de percepción de riesgo fue el más alto percibido para las tres muestras rondando el $80 \%$ de los sujetos que consideran la actividad sexual desprotegida con una pareja casual de alto riesgo. En la historia 2 se plantea la actividad sexual con una pareja "estable" aunque desde hacetan sólotres meses, aquí el nivel de riesgo percibido para la actividad sexual desprotegida es claramente inferior, siendo los chicos y las chicas de la muestra de Barcelona los que perciben menor riesgo en esta actividad. Finalmenteen relación a la historia 3 en la que se plantea una relación de sexo casual fuera de la relación estable que se mantiene con una pareja afectiva desde hace varios años, el nivel de riesgo percibido se acerca al percibido para la actividad sexual desprotegida planteada en la primera historia, ligeramente inferior y sustancialmente superior al percibido en la segunda de las historias planteadas.

Sin embargo, ya que las relaciones afectivo-sexuales delos jóvenes siguen para la mayoría el proceso de "monogamia seriada", que significa mantener relaciones afectivo-sexuales con diversas parejas a largo de la vida, incluso en períodos muy cortos de tiempo, aunque no se simultane en parejas, esto coloca a los jóvenes en una situación de vulnerabilidad no percibida para la infeción $\mathrm{VIH} /$ aids, sobretodo si tenemos en cuenta la dificultad a la hora de informar de una posible infidelidad que con estos trabajos también ha quedado demostrado, sobre todo para aquellos/as que mantienen relaciones sexuales. 
Oraá (1996) comprueba que la mayor percepción de riesgo se dá en las mujeres y en las personas que han mantenido relaciones sexuales. Un dato interesante de este estudio es que el $94 \%$ de quienes mantienen relaciones sexuales manifiestan que se quedarían más tranquilos/as si su pareja les propusiera utilizar el preservativo porque con ello demuestra ser una persona prevenida y responsable. Teniendo en cuenta que los estudios demuestran el obstáculo que el preservativo impone para el desarrollo de la "confianza" percibido como elemento clave para el mantenimiento de la pareja (Lear, 1995), es muy probable que una vez que la pareja se estabilice, el valorarla como "prevenida y responsable" dejará paso a la confianza y por tanto a la innecesaria utilización del preservativo.

En el trabajo de Planes (1993) la mayoría de los jóvenes universitarios/as evaluados creen que sus posibilidades de contagiarse con el HIV se sitúan por debajo de la media cuando se comparan con sus compañeros de curso del mismo sexo y edad, por lo que se comprueba el sesgo optimista independientemente de su nivel de conocimientos en relación a la infección HIV/aids.

Sin embargo, incluso en aquellos sujetos con alta percepción de riesgo al contagio del HIV no utilizan de forma sistemática el preservativo en sus relaciones sexuales. Por tanto la realidad es que los datos nos llevan a concluir que la variable percepción de riesgo surge como variable necesaria pero no suficiente para explicar la conducta preventiva y evitar el contagio del HIV. Es decir, parece que es necesario percibir el riesgo para llevar a cabo una conducta preventiva en la esfera de la sexualidad (van der Pligt et al., 1993) pero percibir el riesgo de contagio del HIV/aids no garantiza que la conducta preventiva se dé (Kelly, Sikkema y Winnett, 1995).

Este hallazgo tiene vital importancia en las campañas desarrolladas para prevenir la infección HIV/aids ya que favorecer la percepción de riesgo no puede ser el único objetivo con el que pueda justificarse el 
cambio de conductay otras variables: a) las normas que imponeel grupo de iguales, b) el incremento de la autoeficacia percibida, y c) el favorecer la identificación del sujeto con las emociones negativas que pueden surgir una vez que la conducta sexual se ha llevado a cabo sin protección, permitiendo que el sentimiento de angustia y de culpa activen el deseo y la motivación del sujeto de llevara cabo una conducta sexual protegida y tranquilizante desde el punto de vista afectivo (van del Pligt et al., 1994, Richar y van der Pligt, 1995) pueden ayudarnos a alcanzar el objetivo de abarcar atodas las "suficientes" y conseguir con ello dar un paso firme en la lucha contra la infección HIV/aids en los más jóvenes.

\section{COMPORTAMIENTO SEXUAL EN LA ADOLESCENCIA}

\section{La actividad sexual coital}

La disminución de la edad de inicio de la primera relación sexual coital y de la frecuencia del coito, aunque varia en función del lugar geográfico al que hagamos referencia, parece constituir una tónica generalizada. Welling y colaboradores (1995) en su trabajo con una muestra de jóvenes ingleses comprueba la reducción de una media de 4 años para las chicas y de tres anos para los chicos en el inicio de la actividad sexual en las pasadas cuatro décadas, con una equiparación entre sexos e identificandose los 17 años como media para ambos.

En los primeros trabajos efectuados en Ourense (Fernández, González, González y López, 1993) la media de edad de chicos universitarios de los primeros cursos con experiencia sexual era del $58 \%$ frente al 38\% de chicas, aunque en el trabajo de Oráa (1996) la actividad sexual apenas alcanza al $30 \%$ (34\% chicos y $24 \%$ chicas). Interesante es el dato que se aporta en este trabajo en el que se 
analizan los motivos que les ha llevado a los jóvenes a no mantener relaciones sexuales: para las chicas se trata sobre todo de "no estar preparada" (42\%) para los chicos de "no haber encontrado la persona adecuada" (43\%).

En España los datos obtenidos en muestras de sujetos escolarizados muestran la menor actividad sexual en relación con otros países europeos o de nuestro entorno socioeconómico, estableciendose en torno al $50 \%$ el nivel de actividad sexual en adolescentes con edades inferiores alos 20 años (ver tabla 2). Y aunque existen diferencias en función del sexo se está produciendo una disminución en los últimos años, lo que en otros países también se constata (Raab, Burns y Scott, 1995). No obstante es más probable todavía que los chicos con experiencia sexual hayan tenido un mayor número de parejas sexuales que las chicas con experiencia, la mayoría en nuestros estudios las han tenido con una sola pareja, siendo más probable que ellas las mantengan dentro de una relación afectiva.

TABLA 2: ACTIVIDAD SEXUAL DE UNIVERSITARIOS/AS.

\begin{tabular}{|l|c|c|c|c|c|c|}
\hline & \multicolumn{2}{|c|}{ Salamanca $^{1}$} & Navarra $^{2}$ & Barcelona $^{3}$ & Piloto $^{4}$ & Ourense $^{5}$ \\
\cline { 2 - 7 } & $\mathbf{1 9 7 7}$ & $\mathbf{1 9 8 7}$ & $\mathbf{1 9 9 5}$ & $\mathbf{1 9 9 6}$ & $\mathbf{1 9 9 6}$ & $\mathbf{1 9 9 7}$ \\
\hline Ninguna & 57,5 & 44,7 & 49,6 & 49,5 & 47,1 & 51,2 \\
\hline Coito con uma pareja & 21,9 & 28,0 & 36,3 & 27,0 & 24,7 & 28,9 \\
\hline Coito con varias parejas & 20,6 & 27,3 & 14,1 & 23,5 & 28,2 & 19,9 \\
\hline & $\begin{array}{l}\mathrm{N}=945 \\
\text { chicos }=44 \%\end{array}$ & $\begin{array}{l}\mathrm{N}=407 \\
\text { chicos=39,8\% }\end{array}$ & $\begin{array}{l}\mathrm{N}=763 \\
\text { chicos=48,6\% }\end{array}$ & $\begin{array}{l}\mathrm{N}=468 \\
\text { chicos=26\% }\end{array}$ & $\begin{array}{l}\mathrm{N}=228 \\
\text { chicos=27,6\% }\end{array}$ & $\begin{array}{l}\mathrm{N}=587 \\
\text { chicos }=35,4 \%\end{array}$ \\
\hline
\end{tabular}

1 López, F. (1990).

${ }^{2}$ Garcia, J. L.; Avis, M.; Cobos, F.; Biurrun, A. C.; Eslava, J. L.; Rodrigo, C.; Padilla, M. A. y Tinajas (1995).

${ }^{3}$ Bayés, Pastells y Tuldrá (1996).

${ }_{5}^{4}$ Lameiras (1997).

${ }^{5}$ Lameiras y Failde (1998). 


\section{El uso del preservativo}

El interés por evaluar el estudio del uso del preservativo viene condicionada por constituir el único profiláctico útil para prevenir E.T.S. y especialmente la transmisión de HIV en las relaciones sexuales que se permite el intercambio de semen y fluidos vaginales.

En el trabajo de Fernández, González, González y López (1993) el $30 \%$ de los sujetos sexualmente activos utiliza siempre el preservativo ( $36 \%$ de chicos y $24 \%$ de chicas) frente al $22 \%$ que no lo ha utilizado nunca (10\% de chicos y $32 \%$ de chicas). En relación con las conductas que implican mayor riesgo el $59 \%$ de los/as universitarios/as que han mantenido relaciones sexuales con más de una pareja en el último año no han utilizado preservativo en todas las ocasiones. Y el uso del preservativo en dicho trabajo no guarda relación estadísticamente significativa ni con los conocimientos en torno a la gravedad de la enfermedad ni con los conocimientos en torno a lasvías de transmisión.

En el trabajo de Lanchares, López, Velasco, Doyague y Tejerizo (1992), con una muestra de 236 adolescentes pre-universitarios de Salamanca, comprueba que el preservativo es utilizado por el $46 \%$ de las chicas y el $80 \%$ de los chicos aunque no se especifica la frecuencia de uso. En la tabla 3 se exponen los datos relativos al uso del preservativo en varios trabajos diferenciando claramente el uso sistemático del preservativo, que implica su utilización siempre durante la práctica sexual, frente al uso ocasional y no uso. En estos trabajos se comprueba que el uso sistemático se encuentra en torno al $50 \%$ de los sujetos, siendo los chicos los que agrupan el mayor porcentaje de uso. Datos que convergen con los obtenidos en el trabajo de Oraá (1996) en el que utilizan siempre el preservativo e $147 \%$ de los sujetos de la muestra, siendo utilizado en la primera relación por el $66 \%$, dato que converge claramente com el obtenido por Aláez, Mayorde la Torre, Madrid, Bavín y Melero (1994) en una muestra de sujetos con una 
media de edad de 17años. Por su parte Arnal y Llario (1994) con una muestra de 1.135 jóvenes de las provincias de Valencia y Castellón, solamente el $25 \%$ de los chicos entre 17 y 18 años manifiesta utilizar preservativos en sus relaciones sexuales frente al $28.9 \%$ de las chicas; en el rango de edad de 19 a 24 anos se incrementó su uso al $50 \%$ de los chicos y el $40 \%$ de las chicas.

Un dato a tener en cuenta en el trabajo de Oraá (1996) es que para el $64 \%$ de los sujetos encuestados las relaciones sexuales surgen de forma espontánea y lo que aún es más importante, el 89\% de los sujetos creen que así es como debe ser. $Y$ en el que además se afirma que el $87 \%$ de los sujetos que mantienen relaciones sexuales con penetración no han accedido nunca a un centro de planificación familiar.

Aunque la inmensa mayoría de los jóvenes españoles hoy en día sabe y reconoce el papel protector que frente al HIV/aids tiene el preservativo, el porcentaje de sujetos que lo utiliza es significativamente menor, y aún menor aquellos/as que lo utilizan de forma sistemática, la forma más eficaz de evitar la transmisión sexual del HIV y de otras enfermedades de transmisión sexual.

TABLA 3: USO DEL PRESERVATIVO EN PORCENTAJES.

\begin{tabular}{|l|c|c|c|c|}
\hline \multirow{2}{*}{} & \multicolumn{2}{|c|}{ Chicos } & \multicolumn{2}{c|}{ Chicas } \\
\cline { 2 - 5 } & Ourense $^{1}$ & Barcelona $^{2}$ & Ourense & Barcelona \\
\hline Siempre & 63 & 45 & 57 & 47 \\
\hline Ocasionalmente & 22 & 43 & 27 & 42 \\
\hline Nunca & 15 & 12 & 16 & 11 \\
\hline
\end{tabular}

${ }^{1}$ Lameiras y Failde (en prensa).

${ }^{2}$ Bayés, Paslells y Tuldrá (1996). 


\section{El uso de otros métodos anticonceptivos}

Enel trabajo de Lameiras y González (1993) el $73.1 \%$ de los sujetos de la muestra con experiencia sexual afirman utilizar algún método anticonceptivo frente al $11.2 \%$ que reconoce no haber utilizado ninguno. Son los chicos los que afirman utilizaren mayor porcentaje anticonceptivos debidos al mayor uso que éstos hacen del preservativo frente a las chicas.

Al evaluar el uso de los métodos anticonceptivos, exceptuando el uso del preservativo al que ya se ha aludido y que es el más utilizado, la píldora es el método anticonceptivo más frecuente que agrupa al $36.9 \%$ de las chicas y es utilizado por el $15 \%$ de las parejas sexuales de los chicos. Seguido del "coitus interruptus" al que recurren el $17.5 \%$ de los chicos y el $20 \%$ de las chicas (Lameiras, 1997a).

En el mismo sentido se manifiestan los sujetos de la muestra del trabajo de Lameiras y Failde (en prensa) que dicen utilizar la píldora el $34.6 \%$ de los chicos y el $12.6 \%$ de las parejas sexuales de los chicos, y el"coitus interruptus" el $11.7 \%$ de los chicos y el $23.7 \%$ de las chicas. En el trabajo de Oraá (1996) en una de cada tres de las primeras relaciones sexuales existe riesgo de embarazo o ETS por no utilizar ningún método (13\%) o uno ineficaz (20\% "coitus interruptus'). En el trabajo de Lanchares, Lopez, Velasco, Doyague y Tejerizo (1992) el $10 \%$ de las chicas utilizan la píldora anticonceptiva y el $12 \%$ "coitus interruptus" junto al $14 . \%$ de los chicos.

\section{CONCLUSIONES}

El incremento de la actividad sexual y la disminución en la edad de inicio, junto a una tendencia en la equiparación entre sexos, el incremento en el nivel de conocimientos, especialmente en relación a la infección HIV/aids, las actitudes más favorables hacia la sexualidad y los/as enfermos de aids y el mayor uso del preservativo - aunque 
no suficiente, sobre todo en las relaciones consideradas "estables", con una pareja afectiva - y la baja percepción de riesgo constituyen las principales conclusiones que el estudio sobre la sexualidad en los jóvenes en España podemos hacer. La necesidad de una adecuada educación sexual y formación de los jóvenes en el ámbito de la sexualidad para favorecer una actividad sexual satisfactoria y libre de riesgos - avalada por el incremento de contagios heterosexuales por HIV en los últimos años - ha dirigido hacia ésta las principales estrategias de prevención auspiciadas por la OMS.

En España la educación sexual se contempla dentro de un sistema de transversalidad que insta a los/as docentes a incluir dichos contenidos impregnando el curriculum de las distintas asignaturas que se imparten en todos los niveles educativos y que "constituyendo una responsabilidad de toda la comunidad educativa, especialmente del equipo docente... han de estar presentes en el proyecto educativo de centro, en el proyecto curricular de etapa y en las programaciones que realiza el profesorado" (Nieda, 1992, prólogo). Así la educación sexual se enmarca en un programa más amplio de "educación para la salud" cuyos objetivos suponen "que los alumnos desarrollen hábitos y costumbres sanos, que los valoren como uno de los aspectos básicos de la calidad de vida y que rehacen las pautas de comportamiento que no conducen a la adquisición de un bienestar físico y mental" (Nieda, 1992, p. 10).

Pero, a pesar de su indudable atractivo e incuestionable justificación científica, la transversalidad en España no ha sido bien aceptada por los especialistas quienes se pronuncian al respecto y aluden a la "falacia" en que casi siempre se convierte o cuando no en una forma abierta de negar la sexualidad (López, 1990b). Por su parte Font (1996, p. 161) defiende que "estamos todavía muy lejos de los mínimos aceptables (en educación sexual) y que el conjunto de los programas y de las intervenciones carece de suficiente peso específico". 
Ante este estado de la cuestión, exceptuando algunos buenos ejemplos de educación sexual en la escuela como la que lleva a cabo el grupo Harimaguada en Tenerife (Islas Canarias), la demanda social de intervenciónen el ámbito de la sexualidad, que puede ayudar a reducir los riesgos vinculados a la actividad sexual desprotegida han favorecido la proliteración de programas que mayoritariamente desde un modelo preventivo se han dirigido a adolescentes, aproximando la intervención a la edad en la que se desencadena mayoritariamente la actividad sexual, siendo el aspecto más abordado la "prevención de la infección HIV/aids.

Pero ha de ser a través de un modelo integrador (Barragan, 1996, p. 20) en el que se "defiende una visión más amplia de la sexualidad humana, entendida como una forma de desarrollo integral, aceptando cualquier orientación sexual, amplio uso de métodos anticonceptivos y, por supuesto, la existencia explícita de la sexualidad en todas las etapas de la vida" amparado en el modelo liberal capitalista, el que necesariamente aglutine los esfuerzos educativos/ formativos para conseguir personas plenamente desarrolladas en la esfera psicosexual, con la que obtener satisfacción, vía de comunicación interpersonal y de transmisión de afectos y ternura, vivida por tanto como fuente de realización personal, y no sesgar hacia la perspectiva de los riesgos vinculados a su ejecución toda su potencialidad.

Solamente canalizando nuestro esfuerzo hacia campañas informativo/educativas que permitan aportar una información amplia y veraz, despojada de mitos y tabúes, para evitar que se viva la sexualidad de forma atormentada y culpabilizada, al mismo tiempo que se estimula la responsabilidad y la adecuada valoración de los riesgos, permitiendo un comportamiento sexual sano y seguro, el objetivo de una juventud con una actividad sexual satisfactoria y saludable será una realidad para nuestros jóvenes y adultos. 


\section{BIBLIOGRAFÍA}

ALÁEZ, M. F. et al. El comportamiento sexual y anticonceptivo de los adolescentes: evaluación de necesidades en el contexto de un programa de salud (Programa Joven). Clínica y Salud, v. 5, n. 1, p. 41-68, 1994.

ARNAL, A. B.; GIL LLARIO, M. D. Salud sexual (I): análisis del comportamiento sexual de adolescentes, jóvenes y adultos en la comunidad valenciana. Análisis y Modificación de Conducta, v. 20, n. 69, p. 111-138, 1994.

BAIRD, T. L. Mexican adolescent sexuality: attitudes, knowledge and sources of information. Hispanic Journal of Behavioral Sciences, v. 15, n. 3, p. 402-417, 1993.

BARRAGAN, F. La educación afectlva y sexual en Andalucía: la evaluación cualitativa de programas. Sevilla/Málaga: Instituto Andaluz de la Mujer; Consejería de la Presidencia, 1996.

BAYÉS, R.; PASTELLS, S.; TULDRÁ, A. Percepción de riesgo de transmisión del virus de inmunodeficiencia humana $(\mathrm{VIH})$ en estudiantes universitarios. Cuadernos de Medicina Psicosomática y Psiquiatria de Enlace, n. 33, p. 22-27, 1995. . Percepción de riesgo de transmisión del virus de inmunodeficiencia humana $(\mathrm{VIH})$ en estudiantes universitarios. Cuadernos de Medicina Psicosomática y Psiquiatría de Enlace, n. 39, p. 24-31, 1996.

BIASCO, F.; TAYLOR, R. College students' attitudes toward aids. College Student Journal, v. 25, n. 3, p. 398-400, 1991.

BYRNE, D. Sex without contraception. In: BYRNE, D.; FISHER, W. A. (Eds.). Adolescents, sex and contraception. Hillsdale: Lawrence Erlbaum Associates, 1983. p. 3-31.

CARPINTERO, E.; FUERTES, A. Validación de la versión castellana del "Sexual Opinion Survey" (SOS). Cuadernos de Medicina Pslcosomática, n. 31, p. 52-62, 1994. 
DESOUSA, E. R.; HUTZ, C. Responses toward sexual stimuli in Brazil as a function of one's gender role identity and sex. Revista Interamericana de Psicología, v. 29, n. 1, p. 13-21, 1995.

EAGLY, A. M.; CHAIKEN, S. The psychology of attitudes. San Diego: Harcourt Brace Janovich, 1993.

EISER, J. R. (Ed.) Social psychology: attitudes, cognition, and social behavior. Cambridge: Cambridge University Press, 1986. (traducido en Ed. Pirámide, 1989).

ERIKSON, E. Identity: youth and crisis. New York: Norton, 1968.

FAILDE, J. M. G.; GONZÁLEZ, M. L. Los adolescentes ante el sida. Conocimientos y actitudes. In: González, M. L. (Eds.). Adolescencia y Sida. Salamanca: KADMOS, 1993, p. 95-114.

FERNÁNDEZ, A. G. et al. Los universitarios ante el sida. Conocimientos, actitudes y prácticas de riesgo. In: González, M. L. (Ed.). Adolescencia y Sida. Salamanca: KADMOS, 1993, p. 159-188.

FISHER. W. A. et al. Erotophobia-Erotophilia as a dimension of personality. The Journal of Sex Research, v.25, n. 1, p. 123-151, 1988.

FONT, P. Educación afectiva e sexual na educación primaria e secundaria. In: III Xornadas de Educación para a Saúde no Ensino. Santiago: Consellería de Sanidade e Servicios Sociais, 1996. p. 161-176.

GARCIA, J. L. et al. Conductas sexuales de riesgo en universitarios/as: unestudio en la Universidad pública de Navarra. Cuadernos de Medicina Psicosomática y psiquiatria de Enlace. n. 36, p. 48-60, 1995.

GONZALEZ-SAIZ, F.; ORTEGA-MARLASCA, M.; SALVADORL. Evaluación de las actitudes ante el sida en población adolescente. Cuadernos de Medicina Psicosomática y psiquiatría de Enlace, n. 31, p. 26-39, 1994.

GREENWALD, A. G. Why are attitudes important. In: PRATKANIS, A. R.; BRECKLER, Y. S. J.; GREENWLAD, A. G. (Eds.). Attitude Structure and function. Hillsdale, New Jersey: Lawrence Erlbaum Associates, 1989. p. $1-10$. 
HALPERN, C. T.; UDRY, J. R. Restosterone and religiosity as preditors of sexual attitudes and activity among adolescent males: a biosocial model. Journal of Bisoclal Science, v. 26, n. 2, p. 217-234, 1994.

KAPLAN, H. S. La nueva terapia sexual. Madrid: Alianza Editorial, 1978. (orig. 1974).

. Transtornos del deseo sexual. Barcelona: Grijalbo, 1982. (orig. 1979).

KATZ, D. The functional approach to the study of attitudes. Public Opinion Quarterly, n. 24, p. 163-214, 1960.

KELLY, J. A; SIKKEMA, K. J.; WINETI, A. A Factors predicting continued high-risk behavior among gay men in small cities: Psychological, behaviors and demographic characteristics related to unsafe sex. Journal of Consulting and Clinical Psychology, n. 63, p. 101-102, 1995.

KINSEY,A C. Sexual behavior In the human female. Filadelphia: Samiders, 1948.

Sexual behavior In the human male. Filadelphia: Samiders, 1953.

LAMEIRAS,M. F.;GONZÁLEZ,M. L. Adolescencia y sexo. In:GONZÁLEZ, M. L. (Ed.). Adolescencia y Sida. Salamanca: KADMOS, 1993. p. 115-138.

Sexualidad y salud en jóvenes universitarios/as: actitudes, actividad sexualy percepción de riesgo de la transmisión del VIH en un estudio piloto. Cuadernos de Medicina Pslco-somática y Psiquiatria de Enlace, n. 42, 1997a.

Sexualidad en adolescentes escolarlzados: un estudio piloto. Comunicación presentada al II Congreso de Educación para la Salud, Sevilla, 1997b.

GONZALEZ, M. F. Aplicación de la escala de erotofobia-erotofilia (Sexual Opinion Survey, SOS) para evaluar las actitudes sexuales en una muestra de universitarios/as. Actas del II Congreso de Psicopedagogia. Braga, Portugal, 1996. p. 307-320.

; FAILDE, J. M. G. Sexualidad y salud en jóvenes universitarios/as: actitudes, actividad sexual y percepción de riesgo de la transmisión 
heterosexual del VIH. Análisis y Modificaclón de Conducta, v. 93, n. 24, p. 27-64, 1998.

; NAVAS, M. D. Los jóvenes ante la sexualidad: evaluación de conocimientos, actitudes y comportamientos. Comunicación presentada al Congreso Nacional de Seisida en Valencia, 1997.

LANCHARES, J. L. et al. Contracepción en la adolescencia. Encuesta a 240 alumnos de un I.N.B. de Salamanca. Cuadernos de Medicina Psicosomática y Psiquiatria de Enlace, n. 22, p. 38-44, 1992.

LEAR, D. Sexual communication in the age of aids: the construction of risk and trust among young adults. Social Science and Medicine, v. 41, n. 9, p. 1.311-1.323, 1995.

LÉVY, J. J.; PEREZ, E.; FRIGAULT, L. A. Los escenarios sexuales de los adolescentes de Oaxaca (México). Cuadernos de Medicina Psicosomátlca y Psiquiatria de Enlace, n. 36, p. 40-47,1995.

LIKERT, A. A technique for the measurement of attitudes. Archives of Psychology, n. 140, p. 1-55, 1932.

LÓPEZ, F. La sexualidad de los universitarios: un estudio comparativo (19771987). Revista de Sexologia, n. 35, p. 1-12, 1990a.

Educación sexual. Madrid: Fundación Universidad Empresa, 1990b.

; FUERTES, A. Para comprender la sexualldad. Navarra: Verbo Divino, 1990.

et al. Actitudes y comportamientos sexuales frente al sida en un grupo de estudiantes espaiioles: estudio preliminar. Cuadernos de Medicina Psicosomática y Psiquiatria de Enlace, n. 25, p. 34-40, 1993.

MASTERS,M.; JOHNSON, V. Respuesta sexual humana. Buenos Aires: Interamericana, 1976 (orig. 1966).

. Incompatibilidad sexual humana. Buenos Aires: Interamericana, 1976 (orig. 1970).

MONCADA, A. La adolescencia forzosa. Barcelona: Gedisa, 1979.

NIEDA, J. Educación para la salud. Madrid: Ministerio de Educación y Ciencia, 1992. 
O.M.S. Pregnancy and abortlon In adolescent. Report on a WHO meeting: World Health Organization. Genéve, 1975.

The child and the adolescent in society. Report on a WHO conference. Regional Office for Europe. Copenhagen: World Health Organization, 1979.

ORAÁ, R. Hábitos sexuales y conducta preventiva de los/as jóvenes de la Rioja. Cuadernos de Medicina Psicosomática y Psiquiatria de Enlace, n. 40, p. 51-61, 1996.

OSGOOD, C. E.; SUCI, G. J.; TANNENBAUM, P. M. The measurement of meaning. Urbano: University of Illinois Press, 1957.

PETTY, R. E.; WEGENER, D. T.; FABRIGAR, L. R. Attitude and attitude change. Annual Review of Psychology, n. 48, p. 609-647, 1997.

PIAGET, J. The moral judgement of the child. Harmondsworth: Penguin, 1932.

PLANES, P. M. Percepción del riesgo personal de sufrir el sida en estudiantes universitarios: implicaciones respecto a la adopción de comportamientos preventivos. Análisis y Modificación de Conducta, v. 19, p. 68, p. 845858, 1993.

PLIGT, J. et al. Perceived risk of aids: unrealistic optimism and self-protective action. In: PRVOR; REEDER, G. D. (Eds.). The social psychology of HIV infection. Hillsdale: Erlbaum, 1993. p. 39-58.

PRATKANIS, A. R; BRECKLER, S. J.; GREENWALD, A. G. (Eds.). Attitude structure and function. Hillsdale, New Jersey: Lawrence Erlbaun Associates, 1989.

RICHARD, R; PLIGT, J.; VRIES, N. Anticipated affective reactions and prevention of aids. The British Psychological Society, n. 34, p. 9-21, 1995.

RAAB, G. M.; BURUS, S. M.; SCOTT, G. HIV prevalence and risk factors in university students. AIDS, n. 9, p.191-197, 1995.

SMITH, M. B.; BRUNER, J. S.; WHITE, R. W. Opinions and Personality, New York: Wiley, 1956.

URZELAV, A. et al. Prevención del sida: consideraciones sobre un programa realizado con 12.000 adolescentes. JANO XL, n. 940, p. 893-899, 1991. 
WELLINGS, K. et al. Provision of sex education and early sexual experience: the relation examined. British Medical Journal, n. 311, p. 417-20, 1995.

WHITE, L. A. et al. Development and validation of a measure of affective orientation to erotic stimuli: the sexual opinion survey. Paper presented at the meeting of the Midwestern Psychological Association. Chicago, 1977. 